\title{
A HYDRODYNAMICAL STUDY OF FRAGMENTING GAS CLOUDS*
}

\author{
JOE MONAGHAN ${ }^{1}$ and JOHN LATTANZIO ${ }^{1,2}$ \\ ${ }^{1}$ Department of Mathematics, Monash University, \\ Clayton, Victoria, 3168, AUSTRALIA. \\ ${ }^{2}$ Institute of Geophysics and Planetary Physics, L-413, \\ L.L.N.L., P.O. Box 808, Livermore, Ca., 94550, USA.
}

\begin{abstract}
We present 3-D hydrodynamical calculations of collapsing rotating gas clouds, with molecular cooling. We find that cooling significantly inreases the number of fragments.
\end{abstract}

\section{The Fragmentation Calculations}

The details of fragmentation are not well understood. We have used SPH to study the collapse of rotating gas clouds. We have recently modified our code to accurately : olve for the gravitational potential in disk systems, and have added cooling from $\mathrm{H}_{2}$ and $\mathrm{CO}$ molecules (taken from Hollenbach and McKee 1979). Details may be found in Monaghan and Lattanzio (1990).

We study the collapse of a $10^{4} M_{\odot}$ gas spheres, with initial radius $12.6 \mathrm{pc}$ and temperature 70K. The density is uniform on average, with perturbations of standard deviation $14 \%$. The cloud has $\alpha=E_{t h} /\left|E_{g}\right|=0.30$. Uniform rotation is added, with $\beta=E_{\text {rot }} /\left|E_{g}\right|=0.47$. We present two calculations, one isothermal and one with molecular cooling. Both have the same field of density perturbations.

Figure 1 shows the particles projected onto the equatorial plane for the isothermal evolution. Initially the gas rapidly collapses down the rotation axis and forms a thin disc. We see the development of an asymmetric mass distribution which finally grows into a binary. We note that this is very similar to the SLING amplification mechanism proposed by Adams et al. (1989).

Figure 2 shows the effect of molecular cooling. A ring forms which then fragments into 5 major condensations. This ring bears a remarkable similarity to the W49A star formation region (Welch et al. 1987). For further details see Lattanzio et al. (in this volume).

* Work performed under the auspices of the U.S. Department of Energy by the Lawrence Livermore National Laboratory under contract No. W-7405-ENG-48. 


\section{Summary}

The fragmentation of clouds depends on the equation of state. Isothermal clouds, if they fragment, do so into fewer lumps than when cooling is included. The number of fragments does not scale with temperature in the same way as the Jeans mass.
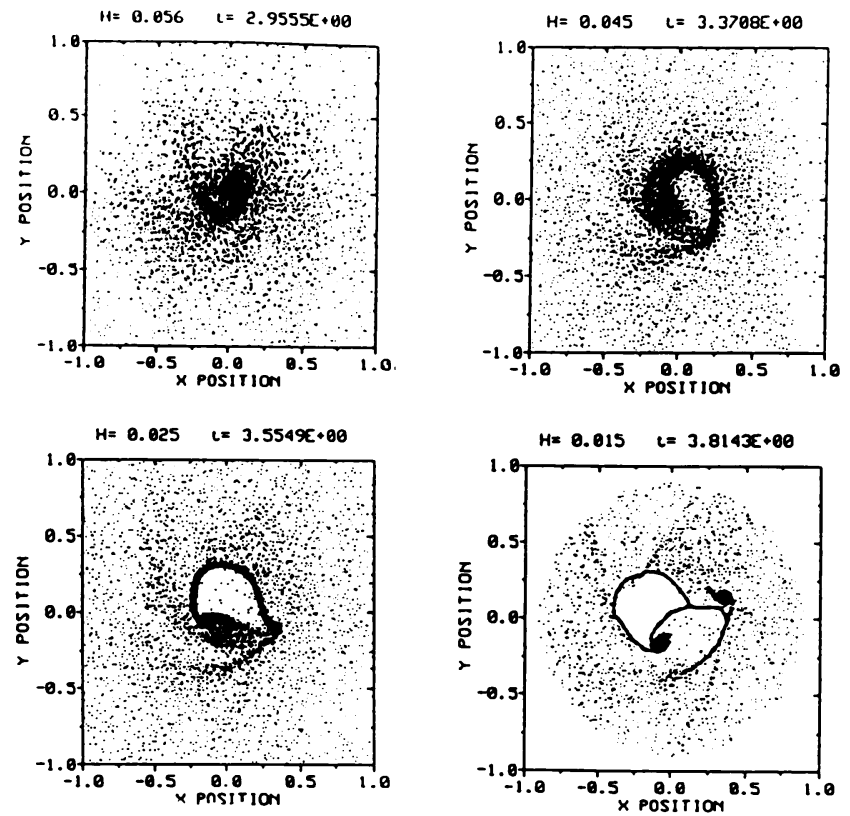

Figure 1: Evolution of the isothermal sequence. Times are in units of the initial free-fall time.
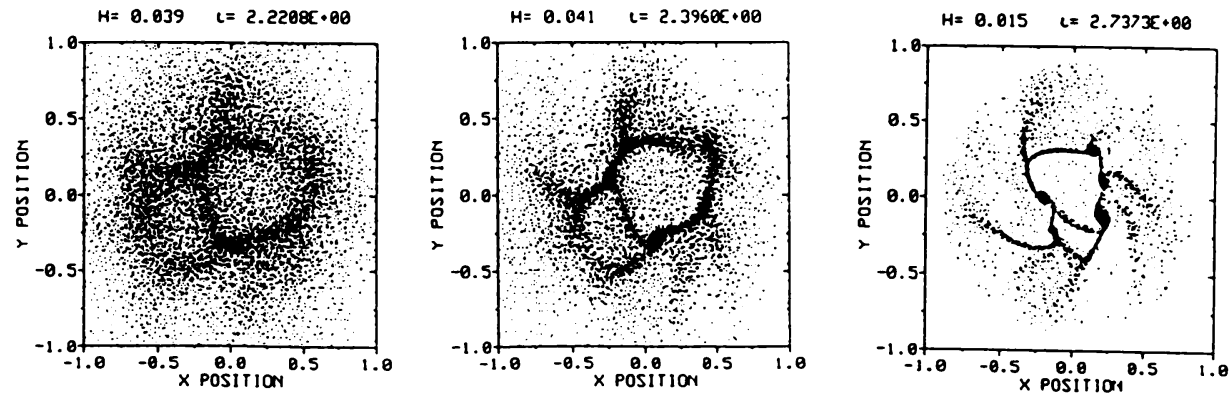

Figure 2: Evolution of the cooling sequence. Times are in units of the initial free-fall time.

\section{References}

Hollenbach, D. and McKee, C., 1979, Astrophys. J. Suppl. Ser., 41, 555.

Monaghan, J. J., and Lattanzio, J. C., 1990, Astrophys. J., submitted.

Adams, F. C., Ruden, S. P., and Shu, F. H., 1989, Astrophys. J., 347, 959.

Welch, W. J., et al,1987, Science, 238, 1550. 